\title{
Candida species in the urinary tract: is it a fungal infection or not?
}

\author{
Vanessa Dias*,1 (iD \\ ${ }^{1}$ Department of Parasitology, Microbiology and Immunology Federal University of Juiz de Fora - UFJF Rua José Lourenço Kelmer, \\ S/n, São Pedro, Juiz de Fora, MG 36036 900, Brazil \\ *Author for correspondence: +55 322101 3213; vancdias2@hotmail.com
}

\begin{abstract}
' the correct identification of Candida species, as well as the in vitro determination of susceptibility profile to the main antifungal agents of medical interest is of utmost importance for therapeutic success."
\end{abstract}

First draft submitted: 18 September 2019; Accepted for publication: 28 January 2020; Published online: 17 February 2020

Keywords: candiduria $\bullet$ fungal infection $\bullet$ urinary tract

Candida species are considered opportunistic pathogens. However, these microorganisms are normally found in the human body. Infections resulting from Candida albicans and non-albicans species have increased significantly in the last decade and are among the leading causes of nosocomial infections, which result in serious public health problems [1].

The finding of Candida species in urine samples (candiduria) represents a diagnostic and therapeutic challenge for physicians working in both primary care or infectious diseases, as well as intensive medicine and surgery [2]. The presence of Candida species in the urine may represent several conditions that require careful interpretation of the report, ranging from sample contamination to urinary tract infections, including disseminated candidiasis $[1,2]$.

Urinary tract infection (UTI) is one of the most commonly diagnosed infections in both hospital and community settings [2-4]. Fungi and bacteria can be etiologic agents of UTI [4]. There is evidence indicating a decrease in the percentage of UTI caused by Escherichia coli, Proteus and Pseudomonas species and an increase in the percentage of UTI caused by fungi, especially Candida species and especially in critically ill patients [4-6].

A recent study [1] demonstrated the occurrence of Candida spp. simultaneously with another urinary tract pathogens (E. coli and Enterococcus faecalis) or two different fungi, type C. albicans and another Candida species in urine samples. However, the frequency of observation of yeast-yeast or yeast-bacteria co-isolation was low: $4.28 \%$ for community patients and $8.33 \%$ for hospitalized patients.

Although C. albicans is the most commonly reported species in urine culture, other species such as Candida glabrata, Candida parapsilosis, Candida tropicalis, Candida kefir, Candida lusitanae, Candida guilhermondi and Candida dubliniensis can also be isolated [1,7]. Another species, Candida auris, was recently isolated in the urine of a hospitalized patient with candidemia [8]. C. auris was first identified from external ear canal discharge in 2009 in Japan [9]. Since then, C. auris isolates have been identified on five continents as agents of nosocomial infections. Reported cases are characterized by high mortality and resistance to various antifungal agents, especially fluconazole. In addition, difficulty in microbiological identification at species level, existence of virulence factors (e.g., easy adhesion and colonization to the human body and environment) and rapid global spread, with several reported outbreaks, lead the scientific community to consider $C$. auris as one of the most serious emerging pathogens $[8,9]$.

Risk factors of candiduria (presence of Candida species in urine samples) and Candida urinary tract infections are well established, and include the extremes of age, female sex, diabetes mellitus, prolonged hospitalization, intensive care unit admission, immunosuppressive therapy, recent use of broad-spectrum antibiotics, previous surgery (urological and nonurological), radiation therapy, genitourinary tuberculosis, neutropenia, urinary tract instrumentation, transplantation, abnormalities of the urinary tract and catheterization, among others $[10,11]$. 
The presence of Candida species in the urine may represent contamination, colonization, UTI, or even candidemia. Contamination can often be differentiated from colonization or UTI by obtaining new urine specimens and checking whether Candida's laboratory finding persists. Other diagnostic methods are unnecessary if the second urine sample does not show the presence of fungi [11].

Thus, it is possible to clinically group patients whose urine presents Candida species: patients with asymptomatic candiduria (previously healthy, outpatients); patients with asymptomatic candiduria (predisposed inpatients); patients with symptomatic candiduria (UTI) and clinically unstable patients with candiduria [5].

Once the presence of Candida in the urine is confirmed, careful clinical evaluation should be performed to detect symptoms or signs suggestive of other conditions such as diabetes mellitus, genitourinary structural abnormalities, decreased renal function and metabolic syndromes [5]. For patients with persistent candiduria, treating certain clinical conditions or removing risk factors is usually sufficient to eliminate the presence of Candida in the urine and no antifungal treatment is required. There is no clinical evidence that antifungal therapy has any benefit in the absence of signs and symptoms of UTI [2].

The management of outpatients with candiduria and some predisposing clinical condition is more complicated because the presence of yeast in the urine may reflect an invasive infection that requires antifungal therapy. Candida's laboratory presence in urine specimens may also be a marker of a process requiring urgent intervention, such as treatment of a urological abnormality. Urinary tract imaging in patients with diabetes mellitus or repeat imaging studies in patients with known abnormalities of the urinary system is recommended so that appropriate treatment can be given [5].

The frequency of UTIs caused by Candida spp. has increased considerably in recent years, especially in hospitalized patients. Individuals with UTI and underlying diseases should be treated with appropriate antifungal medications. Importantly, the treatment of candiduria depends on the clinical condition of the patient $[5,12]$.

The possibility of disseminated candidiasis should be considered, especially in hospitalized patients admitted to the intensive care unit who have candiduria. Candidemia is very frequent in this scenario and 46-68\% of patients with candidemia have associated candiduria. Candidemia is found in $5 \%$ of patients in most intensive care units. Thus, most patients with candiduria probably do not progress to disseminated infection [5,7,12].

Symptomatic Candida cystitis, in most cases, responds clinically to fluconazole which remains a highly active antifungal agent against most Candida species, especially C. albicans; besides being well tolerated and low cost. Importantly, some non-albicans Candida species, such as C. glabrata and Candida krusei, exhibit intrinsic resistance to fluconazole. For refractory bladder infections, flucytosine can be considered an excellent therapeutic option. However, the potential toxicity to bone marrow is a major disadvantage for the clinical use of this antifungal. Treatment with $>1$ intravenous dose of amphotericin B is a third therapeutic option, because this drug has prolonged urinary excretion and is considered a potent antifungal compound [2].

Renal parenchymal infection should be treated, in the same way as candidemia, using systemic antifungal agents. For the treatment of upper urinary tract infections due to the retrograde spread of Candida species, that occurs in patients with obstructive uropathy, diabetes mellitus and frequently concomitant bacteriuria, fluconazole is the antifungal agent of choice [2]. Patients with infection caused by fluconazole resistant isolates may require the use of flucytosine or amphotericin B. In addition to using fluconazole for susceptible organisms or another antifungal, Candida prostatitis or epididymitis usually require incision and drainage, especially if there is an abscess, or resection of prostate tissue or orchiectomy for healing and remission of clinical symptoms [13].

The existence of 'balls' or fungal agglomerates in the urinary tract, as well as the presence of invasive medical devices (Foley catheter, stents, among others) will determine the ideal approach to therapy. Such fungal aggregates and necrotic debris usually result from kidney infection. Fluconazole is the antifungal agent of choice for treating this type of infection. However, if nephrostomy tubes are accessible to the renal pelvis, for example, amphotericin $\mathrm{B}$ irrigation becomes an extremely useful adjuvant therapy. Local saline irrigation and streptokinase application can be successfully used to facilitate the breaking and passage of fungal 'balls' through the urinary tract [13]. Removal or placement of new devices of urinary tract instruments such as catheters are helpful [11].

For critically ill patients, candiduria, whether symptomatic or asymptomatic, should be considered a precursor of disseminated candidiasis, since the kidney is the primary site of candidemia in approximately $80 \%$ of patients. Thus, finding Candida species in the urine may be the only clue that the patient has a severe infection. In these cases, systemic therapy with fluconazole or another azole derivative is advised. An echinocandin, such as caspofungin antifungal, is preferred if the patient has had recent exposure to fluconazole, which is the drug of choice [5,13]. For 
these severely ill patients, antifungal drug prophylaxis appears to be warranted given the additional risk factors for invasive candidiasis [11].

A correct diagnosis will provide treatment appropriate for the patient, besides representing an improvement in mortality rates resulting from these infections. Thus, the correct identification of Candida species, as well as the in vitro determination of susceptibility profile to the main antifungal agents of medical interest is of utmost importance for therapeutic success. Therefore, standardization of conduct adopted by clinicians and laboratory technicians is essential to ensure proper diagnosis, correct treatment and improved quality of life for critically ill patients.

\section{Financial \& competing interests disclosure}

The author has no relevant affiliation or financial involvement with any organization or entity with a financial interest in or financial conflict with the subject matter or materials discussed in the manuscript. This includes employment, consultancies, honoraria, stock ownership or options, expert testimony, grants or patents received or pending, or royalties.

No writing assistance was utilized in the production of this manuscript.

\section{References}

1. Gajdács M, Dóczi I, Ábrók M, Lázár A, Burián K. Epidemiology of candiduria and Candida urinary tract infections in inpatients and outpatients: results from a 10-year retrospective survey. Cent. European J. Urol. 72(2), 209-214 (2019).

2. Fisher JF. Candida urinary tract infections - epidemiology, pathogenesis, diagnosis and treatment: executive summary. Clin. Infect. Dis. 52(Suppl. 6), S429-S432 (2011).

3. Behzadi P, Behzadi E, Ranjbar R. Urinary tract infections and Candida albicans. Cent. European J. Urol. 68(1), 96-101 (2015).

4. Bongomin F, Gago S, Oladele R, Denning DW. Global and multi-national prevalence of fungal diseases - estimate precision. J. Fungi. 3(4), 57-62 (2017).

5. Fisher JF, Sobel JD, Kauffman CA, Newman CA. Candida urinary tract infections: treatment. Clin. Infect. Dis. 52(Suppl. 6), S457-S466 (2011).

6. Gharanfoli A, Mahmoudi E, Torabizadeh R, Katiraee F, Faraji S. Isolation, characterization and molecular identification of Candida species from urinary tract infections. Curr. Med. Mycol. 5(2), 33-36 (2019).

7. Fazeli A, Kordbacheh P, Nazari A et al. Candiduria in hospitalized patients and identification of isolated Candida species by morphological and molecular methods in Ilam, Iran. Iran J. Public Health. 48(1), 156-161 (2019).

8. Biagi MJ, Wiederhold NP, Gibas C et al. Development of high-level echinocandin resistance in a patient with recurrent Candida auris candidemia secondary to chronic candiduria. Open Forum Infect. Dis. 6(7), ofz262 (2019).

9. Iguchi S, Itakura Y, Yoshida A et al. Candidaauris: a pathogen difficult to identify, treat and eradicate and its characteristics in Japanese strains. J. Infect. Chemother. 25(10), 743-749 (2019).

10. Sobel JD, Fisher JF, Kauffman CA, Newman CA. Candida urinary tract infections-epidemiology. Clin. Infect. Dis. 52(Suppl. 6), S433-S436 (2011).

11. Hollenbach E. To treat or not to treat - critically ill patients with candiduria. Mycoses. 51(Suppl. 2), 12-24 (2008).

12. Khairat SM, Sayed SM, Nabih M, Soliman NS, Hassan YM. Prevalence of Candida blood stream infections among children in tertiary care hospital: detection of species and antifungal susceptibility. Infect. Drug Res. 12, 2409-2416 (2019).

13. Pappas PG, Kauffman CA, Andes D et al. Clinical practice guidelines for the management of candidiasis: 2009 update by the Infectious Diseases Society of America. Clin. Infect. Dis. 48(5), 503-535 (2009).

14. Sousa IA, Braoios A, Santos TG et al. Candiduria in adults and children: prevalence and antifungal susceptibility in outpatient of Jataí-GO. J. Bras. Patol. Med. Lab. 50(4), 259-264 (2014). 
